\title{
Arrangements of equal minors in the positive Grassmannian
}

\author{
Miriam Farber|k and Alexander Postnikov"t \\ Department of Mathematics, Massachusetts Institute of Technology, Cambridge MA 02139, USA
}

\begin{abstract}
We discuss arrangements of equal minors in totally positive matrices. More precisely, we would like to investigate the structure of possible equalities and inequalities between the minors. We show that arrangements of equals minors of largest value are in bijection with sorted sets, which earlier appeared in the context of alcoved polytopes and Gröbner bases. Maximal arrangements of this form correspond to simplices of the alcoved triangulation of the hypersimplex; and the number of such arrangements equals the Eulerian number. On the other hand, we conjecture and prove in many cases that arrangements of equal minors of smallest value are exactly the weakly separated sets. Weakly separated sets, originally introduced by Leclerc and Zelevinsky, are closely related to the positive Grassmannian and the associated cluster algebra.
\end{abstract}

Résumé. Il s'agit des arrangements des mineurs égaux dans les matrices totalement positives. Plus précisément, nous aimerions étudier la structure des égalités et inégalités possibles entre les mineurs. Nous montrons que les arrangements des mineurs égaux de plus grande valeur sont en bijection avec les ensembles triés, qui auparavant apparaissaient dans le cadre de polytopes alcôve et bases de Gröbner. Arrangements maximales de ce format correspondent aux simplexes de la triangulation alcôve de la hypersimplex, et le nombre de ces arrangements est égal au nombre eulérien. D'autre part, nous conjecturons et prouvons dans des cas nombreux que les arrangements des mineurs égaux de plus petite valeur sont notamment les ensembles faiblement séparés. Ces ensembles faiblement séparés, initialement introduites par Leclerc et Zelevinsky, sont liés à la Grassmannienne positive et l'algèbre cluster.

Keywords: Totally positive matrices, minors, the positive Grassmannian, Plücker coordinates, matrix completion problem, weakly separated sets, cluster algebras, plabic graphs, sorted sets, triangulations, thrackles, alcoved polytopes, affine Coxeter arrangements, hypersimplices, Eulerian numbers, Gröbner bases, Schur positivity.

\section{Introduction}

In this paper, we investigate possible equalities and inequalities between minors of totally positive matrices. This study turned out to be intimately related to the combinatorics of the positive Grassmannian $[\overline{\mathrm{PO}}]$, weakly separated sets [LZ, OPS], cluster algebras [FZ], alcoved polytopes [LP] and triangulations of hypersimplices, as well as other popular topics of current research in combinatorics.

*Email: mfarberamit.edu.

†Email: apostemath.mit. edu. Supported in part by the NSF grant DMS-1100147. 
One motivation for the study of equal minors came from a variant of the matrix completion problem. The matrix completion problem is the problem of completing missing entries of a partial matrix so that the resulting matrix satisfies a certain property (e.g., it is positive definite or totally positive). Completion problems arise in variety of applications, such as statistics, discrete optimization, data compression, etc.

Recently, the following variant of the completion problem was investigated in [FFJM] and [FRS]. It is well-known that one can "slightly perturb" a totally non-negative matrix and obtain a totally positive matrix (with all strictly positive minors). It is natural to ask how to do this in a "minimal" way. In other words, one would like to find the minimal number of matrix entries that one needs to change in order to get a totally positive matrix. The most degenerate totally non-negative matrix all of whose entries are positive is the matrix filled with all 1's. The above question for this matrix can be equivalently reformulated as follows: What is the maximal number of equal entries in a totally positive matrix? (One can always rescale all equal matrix entries to 1's.) It is then natural to ask about the maximal number of equal minors in a totally positive matrix.

In [FFJM, FRS], it was shown that the maximal number of equal entries in a totally positive $n \times n$ matrix is $\Theta\left(n^{4 / 3}\right)$, and that the maximal number of equal $2 \times 2$-minors in a $2 \times n$-matrix is $\Theta\left(n^{4 / 3}\right)$. It was also shown that the maximal number of equal $k \times k$ minors in a $k \times n$ matrix is $O\left(n^{k-\frac{k}{k+1}}\right)$. The construction is based on the famous result of Szemerédi-Trotter (conjectured by Erdös) about the maximal number of point-line incidences in the plane.

In the present paper, we would like to get a more precise combinatorial description of possible collections of equal minors. In general, this seems to be a very hard problem, which is still far from the complete solution. However, in cases of minors of smallest and largest value, the problem leads to the structures that have a beautiful combinatorial description.

\section{From totally positive matrices to the positive Grassmannian}

A matrix is called totally positive (resp., totally nonnegative) if all its minors, that is, determinants of square submatrices (of all sizes), are positive (resp., nonnegative). The notion of total positivity was introduced by Schoenberg [Sch] and Gantmacher and Krein [GK] in the 1930s. Lusztig [Lu1, Lu2] extended total positivity in the general Lie theoretic setup and defined the positive part for a reductive Lie group $G$ and a generalized partial flag manifold $G / P$.

For $n \geq k \geq 0$, the Grassmannian $G r(k, n)$ (over $\mathbb{R}$ ) is the space of $k$-dimensional linear subspaces in $\mathbb{R}^{n}$. It can be identified with the space of real $k \times n$ matrices of rank $k$ modulo row operations. (The rows of a matrix span a $k$-dimensional subspace in $\mathbb{R}^{n}$.) The maximal $k \times k$ minors of $k \times n$-matrices form projective coordinates on the Grassmannian, called the Plücker coordinates. We will denote the Plücker coordinates by $\Delta_{I}$, where $I$ is a $k$-element subset in $[n]:=\{1, \ldots, n\}$ corresponding to the columns of the maximal minor. These coordinates on $\operatorname{Gr}(k, n)$ are not algebraically independent; they satisfy the Plücker relations.

In [Po], the positive Grassmannian $\mathrm{Gr}^{+}(k, n)$ is described as the subset of the Grassmannian $\operatorname{Gr}(k, n)$ such that all the Plücker coordinates are simultaneously positive: $\Delta_{I}>0$ for all $I$. Similarly, the nonnegative Grassmannian $G r \geq(k, n)$ is defined by the condition $\Delta_{I} \geq 0$ for all $I$. This construction agrees with Lusztig's general theory of total positivity.

The space of totally positive (totally nonnegative) $k \times m$-matrices can be embedded into the positive 
(nonnegative) Grassmannian $G r^{+}(k, n)$ with $n=m+k$, see [이, as follows:

$$
A=\left(a_{i j}\right) \longmapsto\left(\begin{array}{cccccccccc}
1 & 0 & \cdots & 0 & 0 & 0 & (-1)^{k-1} a_{k 1} & (-1)^{k-1} a_{k 2} & \cdots & (-1)^{k-1} a_{k m} \\
\vdots & \vdots & \ddots & \vdots & \vdots & \vdots & \vdots & \vdots & \ddots & \vdots \\
0 & 0 & \cdots & 1 & 0 & 0 & a_{31} & a_{32} & \cdots & a_{3 m} \\
0 & 0 & \cdots & 0 & 1 & 0 & -a_{21} & -a_{22} & \cdots & -a_{2 m} \\
0 & 0 & \cdots & 0 & 0 & 1 & a_{11} & a_{12} & \cdots & a_{1 m}
\end{array}\right)
$$

Under this map, all minors (of all sizes) of the $k \times m$-matrix $A$ are equal to the maximal $k \times k$-minors of the extended $k \times n$ matrix on the right.

This map is actually a bijection between the space of totally positive $k \times m$ matrices and the positive Grassmannian $G r^{+}(k, n)$. However, the nonnegative Grassmannian $G r \geq(k, n)$ is strictly bigger than the space of totally nonnegative $k \times m$-matrices; and it has a more subtle combinatorial structure.

This construction allows us to reformulate questions about equalities and inequalities between minors (of various sizes) in terms of analogous questions for the positive Grassmannian, involving only maximal $k \times k$ minors (the Plücker coordinates). One immediate technical simplification is that, instead of minors with two sets of indices (for rows and columns), we will use the Plücker coordinates $\Delta_{I}$ with one set of column indices $I$. More significantly, the reformulation of the problem in terms of the Grassmannian unveils symmetries which are hidden on the level of matrices.

Indeed, the positive Grassmannian $\operatorname{Gr}^{+}(k, n)$ possesses the cyclic symmetry. Let $\left[v_{1}, \ldots, v_{n}\right]$ denotes a point in $\operatorname{Gr}(k, n)$ given by $n$ column vectors $v_{1}, \ldots, v_{n} \in \mathbb{R}^{k}$. Then the map

$$
\left[v_{1}, \ldots, v_{n}\right] \mapsto\left[(-1)^{k} v_{n}, v_{1}, v_{2}, \ldots, v_{n-1}\right]
$$

preserves the positive Grassmannian $G r^{+}(k, n)$. This defines the action of the cyclic group $\mathbb{Z} / n \mathbb{Z}$ on the positive Grassmannian $G r^{+}(k, n)$.

We will see that all combinatorial structures that appear in our study of the positive Grassmannian and arrangements of equal minors have the cyclic symmetry related to this action of $\mathbb{Z} / n \mathbb{Z}$.

Definition 2.1 Let $\mathcal{I}=\left(\mathcal{I}_{0}, \mathcal{I}_{1}, \ldots, \mathcal{I}_{l}\right)$ be an ordered set-partition of the set $\left(\begin{array}{c}{[n]} \\ k\end{array}\right)$ of all $k$-element subsets in $[n]$. Let us subdivide the nonnegative Grassmannian $G r \geq(k, n)$ into the strata $S_{\mathcal{I}}$ labelled by such ordered set partitions $\mathcal{I}$ and given by the conditions:

1. $\Delta_{I}=0$ for $I \in \mathcal{I}_{0}$,

2. $\Delta_{I}=\Delta_{J}$ if $I, J \in \mathcal{I}_{i}$,

3. $\Delta_{I}<\Delta_{J}$ if $I \in \mathcal{I}_{i}$ and $J \in \mathcal{I}_{j}$ with $i<j$.

An arrangement of minors $i$ s an ordered set-partition $\mathcal{I}$ such that the stratum $S_{\mathcal{I}}$ is not empty.

Problem 2.2 Describe combinatorially all possible arrangements of minors in $G r \geq(k, n)$. Investigate the geometric and combinatorial structure of the above stratification.

For $k=1$, this stratification is equivalent to the subdivision of the linear space $\mathbb{R}^{n}$ by the hyperplanes $x_{i}=x_{j}$, which form the Coxeter arrangement of type A (also known as, the braid arrangement). The structure of the Coxeter arrangement is well studied. Combinatorially, it is equivalent of the face structure of the permutohedron. 
For $k \geq 2$, the above problem seems to be quite nontrivial.

In [Po], we combinatorially described the cell structure of the nonnegative Grassmannian $G r \geq(k, n)$, which is equivalent to the description of possible sets $\mathcal{I}_{0}$. This description already involves quite rich and nontrivial combinatorial structures. We described possible $\mathcal{I}_{0}$ 's in terms of various combinatorial objects: decorated permutations, positroids, L-diagrams, Grassmann necklaces, etc. The above stratification of $G r \geq(k, n)$ is a finer stratification than the positroid stratification studied in [Po]. It should lead to even more interesting combinatorial objects.

In the present paper, we mostly discuss the case of the positive Grassmannian $G_{r}^{+}(k, n)$, that is, we assume that $\mathcal{I}_{0}=\emptyset$. We concentrate on a combinatorial description of possible sets $\mathcal{I}_{1}$ and $\mathcal{I}_{l}$.

Definition 2.3 We will call a possible set $\mathcal{I}_{1}$ an arrangement of smallest minors in $\mathrm{Gr}^{+}(k, n)$. Also we will call a possible set $\mathcal{I}_{l}$ an arrangement of largest minors in $\mathrm{Gr}^{+}(k, n)$. (Here we assume that $\mathcal{I}_{0}=\emptyset$.)

As a warm up, in the next section we discuss the case $k=2$. We will treat the general case in the subsequent sections.

\section{Case $k=2$ : triangulations and thrackles}

In case $k=2$, one can identify 2-element sets $I=\{i, j\}$ that label the Plücker coordinates $\Delta_{I}$ with the edges $(i, j)$ of the complete graph $K_{n}$ on the vertices $1, \ldots, n$. A subset in $\left(\begin{array}{c}{[n]} \\ 2\end{array}\right)$ can be identified with a subgraph $G \subset K_{n}$.

Let us assume that the vertices $1, \ldots, n$ are arranged on the circle in the clockwise order.

We say that two edges $(i, j)$ and $(r, s)$ are non-crossing if the corresponding straight-line chords $(i, j)$ and $(r, s)$ in the circle do not cross each other. For example, the two edges $(1,4)$ and $(2,3)$ are noncrossing; while the edge $(1,3)$ and $(2,4)$ are crossing.

Theorem 3.1 A nonempty subgraph $G \subset K_{n}$ corresponds to an arrangement of smallest minors in $\mathrm{Gr}^{+}(2, n)$ if and only if every pair of edges in $G$ is non-crossing (or they share a common vertex).

Theorem 3.2 A nonempty subgraph $H \subset K_{n}$ corresponds to an arrangement of largest minors in $\mathrm{Gr}^{+}(2, n)$ if and only if every pair of edges in $H$ is crossing (or they share a common vertex).

In one direction, these two theorems easily follow from the 3-term Plücker relation:

$$
\Delta_{13} \Delta_{24}=\Delta_{12} \Delta_{34}+\Delta_{14} \Delta_{23} .
$$

Recall that we assume that all the minors $\Delta_{I}$ are strictly positive. Indeed, if $\Delta_{13}=\Delta_{24}=a$ then some of the minors $\Delta_{12}, \Delta_{23}, \Delta_{34}, \Delta_{14}$ should be strictly less than $a$. Thus the pair of crossing edges $(1,3)$ and $(2,4)$ cannot belong to an arrangement of smallest minors. On the other hand, if, say, $\Delta_{12}=\Delta_{34}=b$, then $\Delta_{13}$ or $\Delta_{24}$ should be strictly greater than $b$. Thus the pair of non-crossing edges $(1,2)$ and $(3,4)$ cannot belong to an arrangement of largest minors. In a similar fashion, we can show that any other pair of crossing (non-crossing) edges cannot occur in an arrangement of smallest (largest) minors.

Maximal subgraphs $G \subset K_{n}$ without crossing edges are exactly triangulations of the $n$-gon. They contain all "boundary" edges $(1,2),(2,3), \ldots,(n-1, n),(n, 1)$ as well as some $n-3$ non-crossing diagonals that subdivide the $n$-gon into triangles. Of course, the number of such triangulations is the famous Catalan number $C_{n-2}=\frac{1}{n-1}\left(\begin{array}{c}2(n-2) \\ n-2\end{array}\right)$. 
Subgraphs $H \subset K_{n}$ such that every pair of edges crosses or shares a vertex are called thrackles (i) Maximal thrackles in $K_{n}$ should have exactly $n$ edges. They are obtained from an odd star by attaching some leaves; see Figures 1 and 3 below. The number of such maximal thrackles is the Eulerian number $A(n-1,1)=2^{n-1}-n$.

Figures 1, 2 and 3 show examples of triangulations of $n$-gons and thrackles.
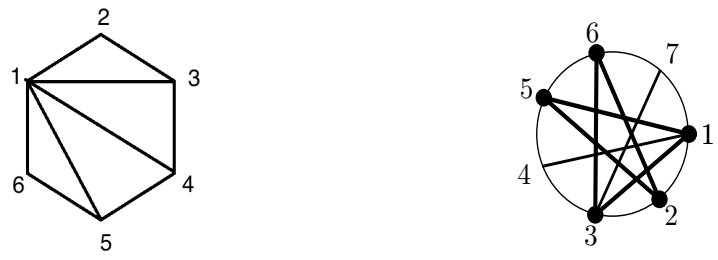

Fig. 1: A triangulation (left) and a thrackle (right). The edges of the triangulation correspond to the arrangement of equal Plücker coordinates $\Delta_{12}=\Delta_{23}=\Delta_{34}=\Delta_{45}=\Delta_{56}=\Delta_{16}=\Delta_{13}=\Delta_{14}=\Delta_{15}$ of smallest value in the positive Grassmannian $\mathrm{Gr}^{+}(2,6)$; while the edges of the thrackle correspond to the arrangement of equal Plücker coordinates $\Delta_{13}=\Delta_{14}=\Delta_{15}=\Delta_{25}=\Delta_{26}=\Delta_{36}=\Delta_{37}$ of largest value in for $G r^{+}(2,7)$. This thrackle is obtained from the 5 -star by adding two leaves.
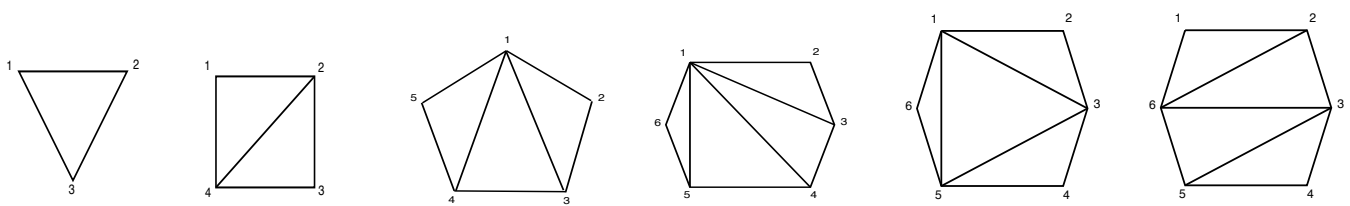

Fig. 2: All triangulations of $n$-gons for $n=3,4,5,6$ (up to rotations and reflections).
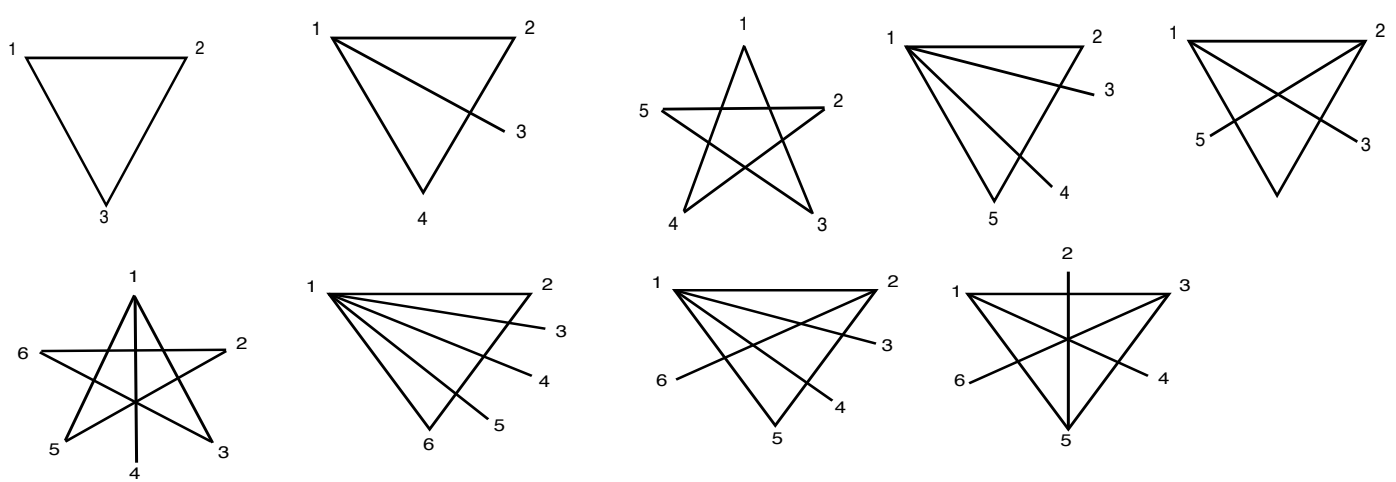

Fig. 3: All maximal thrackles that have 3, 4, 5, or 6 vertices (up to rotations and reflections).

(i) Our thrackles are a special case of Conway's thrackles. The latter are not required to have vertices arranged on a circle. 
Corollary 3.3 Maximal arrangements of smallest minors in $\mathrm{Gr}^{+}(2, n)$ correspond to triangulations of the n-gon. They contain exactly $2 n-3$ minors. The number of such maximal arrangements is the Catalan number $C_{n-2}=\frac{1}{n-1}\left(\begin{array}{c}2(n-2) \\ n-2\end{array}\right)$.

Corollary 3.4 Maximal arrangements of largest minors in $\mathrm{Gr}^{+}(2, n)$ correspond to maximal thrackles in $K_{n}$. They contain exactly $n$ minors. The number of such maximal arrangements is the Eulerian number $A(n-1,1)=2^{n-1}-n$.

\section{Weakly separated sets and sorted sets}

In this section, we show how to extend triangulations and thrackles to the case of general $k$.

As before, we assume that the vertices $1, \ldots, n$ are arranged on the circle in the clockwise order.

Definition 4.1 Two k-element sets $I, J \in\left(\begin{array}{c}{[n]} \\ k\end{array}\right)$ are called weakly separated if their set-theoretic differences $I \backslash J=\left\{a_{1}, \ldots, a_{r}\right\}$ and $J \backslash I=\left\{b_{1}, \ldots, b_{r}\right\}$ are separated from each other by some diagonal in the circle, i.e., $a_{1}<\cdots<a_{s}<b_{1}<\ldots<b_{r}<a_{s+1}<\cdots<a_{r}$ (or the same inequalities with a's and b's switched).

A subset of $\left(\begin{array}{c}{[n]} \\ k\end{array}\right)$ is called weakly separated if every two elements in it are weakly separated.

The notion of weakly separated sets was originally introduced by Leclerc-Zelevinsky [LZ] in the study of quasi-commuting quantum minors.

In [OPS], maximal (by containment) weakly separated subsets in $\left(\begin{array}{c}{[n]} \\ k\end{array}\right)$ were shown to be in bijection with reduced plabic graphs that appear in the study of the positive Grassmannian [Po]. In particular, this construction implies that every maximal weakly separated subset in $\left(\begin{array}{c}{[n]} \\ k\end{array}\right)$ contains exactly $k(n-k)+1$ elements (the Purity Conjecture of Leclerc-Zelevinsky); and that all such maximal weakly subsets can be obtained from each other by a sequence of mutations. A different way to prove the purity conjecture was given by Danilov-Karzanov-Koshevoy [DKK].

Definition 4.2 Two k-element sets $I, J \in\left(\begin{array}{c}{[n]} \\ k\end{array}\right)$ are called sorted if their set-theoretic differences $I \backslash J=$ $\left\{a_{1}, \ldots, a_{r}\right\}$ and $J \backslash I=\left\{b_{1}, \ldots, b_{r}\right\}$ are interlaced on the circle, i.e., $a_{1}<b_{1}<a_{2}<b_{2}<\cdots<$ $a_{r}<b_{r}$ (or the same inequalities with a's and b's switched).

A subset of $\left(\begin{array}{c}{[n]} \\ k\end{array}\right)$ is called sorted if every two elements in it are sorted.

Sorted sets appear in the study of Gröbner bases $[\overline{\mathrm{St}}]$ and in the theory of alcoved polytopes [LP]. According to [LP], any maximal (by containment) sorted subset in $\left(\begin{array}{c}{[n]} \\ k\end{array}\right)$ has exactly $n$ elements. Such subsets were identified with simplices of the alcoved triangulation of the hypersimplex $\Delta_{k, n}$. The number of maximal sorted subsets in $\left(\begin{array}{c}{[n]} \\ k\end{array}\right)$ equals the Eulerian number $A(n-1, k-1)$, that is, the number of permutations $w$ of size $n-1$ with exactly $k-1$ descents, $\operatorname{des}(w)=k-1$. (Recall, that a descent in a permutation $w$ is an index $i$ such that $w(i)>w(i+1)$.) Actually, an explicit bijection between sorted subsets in $\left(\begin{array}{c}{[n]} \\ k\end{array}\right)$ and permutations of size $n-1$ with $k-1$ descents was constructed in [LP].

Here is our main result on arrangements of largest minors.

Theorem 4.3 A subset of $\left(\begin{array}{c}{[n]} \\ k\end{array}\right)$ is an arrangement of largest minors in $\mathrm{Gr}^{+}(k, n)$ if and only if it is a sorted subset. Maximal arrangements of largest minors contain exactly $n$ minors. The number of maximal arrangements of largest minors in $\mathrm{Gr}^{+}(k, n)$ equals the Eulerian number $A(n-1, k-1)$.

Here are our main results and a conjecture on arrangements of smallest minors. 
Theorem 4.4 Any weakly separated subset of $\left(\begin{array}{c}n \\ {[k]}\end{array}\right)$ is an arrangement of smallest minors in $G r^{+}(k, n)$.

Conjecture 4.5 A subset of $\left(\begin{array}{c}{[n]} \\ k\end{array}\right)$ is an arrangement of smallest minors in $\mathrm{Gr}^{+}(k, n)$ if and only if it is a weakly separated subset.

In particular, this conjecture implies that any maximal arrangement of smallest minors in $\mathrm{Gr}^{+}(k, n)$ contains exactly $k(n-k)+1$ minors.

Theorem 4.6 The above conjecture is true for $k=1,2,3$ (and arbitrary $n$ ).

In order to prove the above claims in one direction, we need to show that any two elements $I$ and $J$ in a arrangement of largest (smallest) minors should be sorted (weakly separated). In order to prove the claims in the opposite direction, it is enough to construct, for each sorted (weakly separated) subset, matrices with the corresponding collection of equal largest (smallest) minors.

In the next section we discuss our method of proving that the subsets of equal minors should be sorted or weakly separated. In the subsequent sections we show how to explicitly construct matrices with needed equalities between the minors.

\section{Inequalities for products of minors}

As we mentioned above, in the case $k=2$, in one direction, our results follow from the inequalities for products of minors, like $\Delta_{13} \Delta_{24}>\Delta_{12} \Delta_{34}$. There are analogous inequalities in the general case found by Skandera $[\mathrm{Sk}]$.

Let us reformulate Skandera's result in terms of the Grassmannian and maximal minors. For $I, J \in$ $\left(\begin{array}{c}{[n]} \\ k\end{array}\right)$ and an interval $[a, b]:=\{a, a+1, \ldots, b\} \subset[n]$, define

$$
r(I, J ; a, b)=|(|(I \backslash J) \cap[a, b]|-|(J \backslash I) \cap[a, b]|)| .
$$

Note that the pair $I, J$ is sorted if and only if $r(I, J ; a, b) \leq 1$ for all $a$ and $b$. In a sense, $r(I, J ; a, b)$ is a measure of "unsortedness" of the pair $I, J$.

Theorem 5.1 Skandera [Sk] For $I, J, K, L \in\left(\begin{array}{c}{[n]} \\ k\end{array}\right)$, the products of the Plücker coordinates satisfy the inequality

$$
\Delta_{I} \Delta_{J} \geq \Delta_{K} \Delta_{L}
$$

for all points of the nonnegative Grassmannian $G r \geq(k, n)$, if and only if the multiset union of $I$ and $J$ equals to the multiset union of $K$ and $L$; and, for any interval $[a, b] \subset[n]$, we have

$$
r(I, J ; a, b) \leq r(K, L ; a, b) .
$$

Roughly speaking (maybe very roughly speaking), this theorem says that the product of minors $\Delta_{I} \Delta_{J}$ should be "large" if the pair $I$ and $J$ is sorted; and the product should be "small" if the pair $I$ and $J$ is weakly separated.

For our purposes, we actually need a similar result with strict inequalities $\Delta_{I} \Delta_{J}>\Delta_{K} \Delta_{L}$ for points of the positive Grassmannian $G r^{+}(k, n)$. It can be proved using the same techniques as in [Sk]. This (slightly modified) Skandera's theorem implies the following claim.

For $I, J \in\left(\begin{array}{c}{[n]} \\ k\end{array}\right)$, define their sorting $I^{\prime}, J^{\prime}$ by taking the multiset union $I \cup J=\left\{a_{1} \leq a_{2} \leq \cdots \leq a_{2 k}\right\}$ and setting $I^{\prime}=\left\{a_{1}, a_{3}, \ldots, a_{2 k-1}\right\}$ and $J^{\prime}=\left\{a_{2}, a_{4}, \ldots, a_{2 k}\right\}$. 
Corollary 5.2 Let $I, J \in\left(\begin{array}{c}{[n]} \\ k\end{array}\right)$ be a pair which is not sorted, and let $I^{\prime}, J^{\prime}$ be the sorting of the pair $I, J$. Then we have the strict inequality $\Delta_{I^{\prime}} \Delta_{J^{\prime}}>\Delta_{I} \Delta_{J}$ for points of the positive Grassmannian $G^{+}(k, n)$.

This result implies one direction of Theorem 4.3 Indeed, $I$ and $J$ which are not sorted cannot belong to an arrangement of largest minors. Otherwise, we would have $\Delta_{I}=\Delta_{J}=a$ and the inequality $\Delta_{I^{\prime}} \Delta_{J^{\prime}}>\Delta_{I} \Delta_{J}$ would imply that $\Delta_{I^{\prime}}$ or $\Delta_{J^{\prime}}$ is greater than $a$.

Using similar arguments based on Skandera's theorem we proved the same direction of Conjecture 4.5 for $k=1,2,3$. In these cases, for any product of minors $\Delta_{I} \Delta_{J}$, where $I$ and $J$ are not weakly separated, one can find a Skandera's inequality of the form $\Delta_{I} \Delta_{J}>\Delta_{K} \Delta_{L}$. If $\Delta_{I}=\Delta_{J}=a$, then one the minors $\Delta_{K}$ or $\Delta_{L}$ should be strictly smaller than $a$. Thus a non-weakly-separated pair $I, J$ cannot belong to an arrangement of smallest minors.

However, this approach does not work for $k \geq 4$. The following example shows that the above statement (which is true for $k \leq 3$ ) fails for $k=4$.

Example 5.3 The pair $\{1,5,6,8\},\{2,3,4,7\}$ is not weakly separated. However, there exists a point in $G r^{+}(4,8)$ such that the product of minors $\Delta_{1568} \Delta_{2347}$ is smaller than all other products of complementary minors $\Delta_{I} \Delta_{\bar{I}}$. One concrete example, can be obtained by taking the image of the following totally positive $4 \times 4$ matrix under the embedding $\operatorname{Mat}(4,4) \rightarrow G r(4,8)$ described in Section 2 .

$$
A=\left(\begin{array}{cccc}
1 / 2 & 1 / 2 & 1 / 2 & 1 / 2 \\
1 / 2 & 4097 / 8192 & 1025 / 2048 & 16403 / 32768 \\
1 / 2 & 1025 / 2048 & 2059 / 4096 & 33013 / 65536 \\
1 / 2 & 8233 / 16384 & 4223 / 8192 & 199561 / 131072
\end{array}\right) .
$$

The product $\Delta_{1568} \Delta_{2347}$ in $\operatorname{Gr}(4,8)$ corresponds to the product of minors $\Delta_{4,3} \Delta_{123,124}$ of the $4 \times 4$ matrix A.

This example shows that Skandera's inequalities are not enough to prove Conjecture 4.5 in the general case. In order to prove this conjecture, one might need to find more general inequalities for minors.

Here is a challenge problem about $4 \times 4$ matrices that would imply Conjecture 4.5 for $k=4$.

Problem 5.4 Prove the following claim or construct a counterexample. If two complementary minors $\Delta_{4,3}$ and $\Delta_{123,124}$ of a totally positive $4 \times 4$ matrix are equal to each other, then there is another minor of this matrix which is strictly less than $\Delta_{4,3}=\Delta_{123,124}$.

\section{Cluster algebra on the Grassmannian}

The construction of points in $\mathrm{Gr}^{+}(k, n)$ with maximal arrangements of smallest minors is based on the results of [ [Po,,$\overline{\text { OPS }}]$. The following statement follows from these works.

Theorem 6.1 Any maximal weakly separated subset $S \subset\left(\begin{array}{c}{[n]} \\ k\end{array}\right)$ corresponds to $k(n-k)+1$ algebraically independent Plücker coordinates $\Delta_{I}, I \in S$. Any other Plücker coordinate $\Delta_{J}$ can be uniquely expressed in terms of the $\Delta_{I}, I \in S$, by subtraction-free rational expressions.

This theorem is related to Fomin-Zelevinsky's theory of cluster algebras [FZ]. The Plücker coordinates $\Delta_{I}, I \in S$ form an initial cluster of the cluster algebra associated with the Grassmannian $\operatorname{Gr}(k, n)$. Other maximal weakly separated subsets in $\left(\begin{array}{c}{[n]} \\ k\end{array}\right)$ are obtained from the initial one by a sequence of mutations. This cluster algebra was studied by Scott [Sc] . 
According to Fomin-Zelevinsky's general theory of cluster algebras [FZ], the subtraction-free expressions mentioned in Theorem 6.1 are actually Laurent polynomials (the Laurent phenomenon). FominZelevinsky conjectured and Lee-Schiffler $[\overline{\mathrm{LS}}]$ recently proved (for skew-symmetric cluster algebras) that these Laurent polynomials have positive integer coefficients.

Theorem 6.1 implies that any maximal weakly separated subset $S$ uniquely defines a point $A_{S}$ in the positive Grassmannian $G r^{+}(k, n)$ such that the Plücker coordinates $\Delta_{I}$, for all $I \in S$, are equal to each other. Using Lee-Schiffler's positivity theorem [LS], we deduce that all other Plücker coordinates are strictly greater than the $\Delta_{I}$, for $I \in S$. This proves Theorem 4.4. We can now reformulate Conjecture 4.5.

Conjecture 6.2 Any point in $\mathrm{Gr}^{+}(k, n)$ with a maximal arrangement of smallest equal minors has the form $A_{S}$, for some maximal weakly separated subset $S \subset\left(\begin{array}{c}{[n]} \\ k\end{array}\right)$.

\section{Constructions of matrices for arrangements of largest minors}

In the previous section, we saw that the points in $\mathrm{Gr}^{+}(k, n)$ with a maximal arrangement of smallest equal minors have a very rigid structure. On the other hand, the cardinality of a maximal arrangement of largest minors is $n$, which is much smaller than the cardinality $k(n-k)+1$ of a maximal arrangement of smallest minors. Maximal arrangements of largest minors impose fewer conditions on points of $G r^{+}(k, n)$ and have much more flexible structure. Actually, one can get any maximal arrangement of largest minors from any point of $\mathrm{Gr}^{+}(k, n)$ by the torus action.

The positive torus ${ }^{\text {(ii) }} \mathbb{R}_{>0}^{n}$ acts on the positive Grassmannian $G r^{+}(k, n)$ by rescaling the coordinates in $\mathbb{R}^{n}$. In terms of $k \times n$ matrices this action is given by rescaling the columns of the matrix.

Theorem 7.1 For any point $A$ in $\mathrm{Gr}^{+}(k, n)$ and any maximal sorted subset $S \subset\left(\begin{array}{c}{[n]} \\ k\end{array}\right)$, there is a unique point $A^{\prime}$ of $\mathrm{Gr}^{+}(k, n)$ obtained from $A$ by the torus action (that is, by rescaling the columns of the $k \times n$ matrix A) such that the Plücker coordinates $\Delta_{I}$, for all $I \in S$, are equal to each other. All other Plücker coordinates for the point $A^{\prime}$ are strictly less than the $\Delta_{I}$, for $I \in S$.

The proof of this result is based on geometric techniques of alcoved polytopes and affine Coxeter arrangements developed in [LP].

Let us give some examples of $3 \times n$ matrices $A=\left[v_{1}, v_{2}, \ldots, v_{n}\right]$ with maximal arrangements of largest equal minors. Here $v_{1}, \ldots, v_{n}$ are 3 -vectors. Projectively, we can think about the 3 -vectors $v_{i}$ as points in the (projective) plane. More precisely, let $P \simeq \mathbb{R}^{2}$ be an affine plane in $\mathbb{R}^{3}$ that does not pass through the origin 0 . A point $p$ in the plane $P$ represents the 3 -vector $v$ from the origin 0 to $p$. A collection of points $p_{1}, \ldots, p_{n} \in P$ corresponds to an element $A=\left[v_{1}, \ldots, v_{n}\right]$ of the positive Grassmannian $G r^{+}(3, n)$ if and only if the points $p_{1}, \ldots, p_{n}$ form vertices of a convex $n$-gon (with clockwise order of the $p_{i}$ ).

Let us now assume that the $n$-gon formed by the points $p_{1}, \ldots, p_{n}$ is a regular $n$-gon. Theorem 7.1 implies that it is always possible to uniquely rescale (up to a common factor) the corresponding 3 -vectors by some positive scalars $\lambda_{i}$ in order to get any sorted subset in $\left(\begin{array}{c}{[n]} \\ 3\end{array}\right)$. Geometrically, for a triple $I=$ $\{i, j, r\}$, the minor $\Delta_{I}$ equals the area of the triangle with the vertices $p_{i}, p_{j}, p_{r}$ times the product of the scalar factors $\lambda_{i}, \lambda_{j}, \lambda_{r}$ (times a common factor which can be ignored). We want to make the largest area of such rescaled triangles to repeat as many times as possible.

Figures 4 and 5 show all rescalings of vertices of the regular pentagon and the regular hexagon that give maximal sorted subsets.

(ii) It might be a little strange to call $\mathbb{R}_{>0}^{n}$ the "positive torus", since topologically this contractible set is quite far from been a torus. However, $\mathbb{R}_{>0}^{n}$ is the positive part of the complex torus $(\mathbb{C} \backslash\{0\})^{n}$. 
1

1

$1 \quad 1$ $\phi$

$\phi$

1
1

$\phi$

$\phi^{2} \quad \phi^{2}$

Fig. 4: For the regular pentagon, there are the Eulerian number $A(4,2)=11$ rescalings that give maximal sorted subsets in $\left(\begin{array}{c}{[5]} \\ 3\end{array}\right)$. In the first case, all the scalars $\lambda_{i}$ are 1 . In the second case, the $\lambda_{i}$ are $1,1, \phi, \phi, \phi$ (in the clockwise order). Here $\phi=(1+\sqrt{5}) / 2$ is the golden ratio. (There are 5 rotations of this case.) In the last case, the $\lambda_{i}$ are $1, \phi, \phi^{2}, \phi^{2}, \phi$. (Again, there are 5 rotations.) In total, we get $1+5+5=11$ rescalings.
11
$1 \quad 1$
11
$3 \quad 3$

2

$3 / 2$

$3 / 2$

2

1

$3 / 2$

3

44

$9 / 4 \quad 9 / 4$

32

$1 \quad 3 / 2$

(1)

11

$9 / 4$

$9 / 4$

$3 / 2$

$3 / 2$

$3 / 2$

1

$3 / 2$

$9 / 4$

1

1

$9 / 4 \quad 3 / 2$

11

$3 / 2$

$3 / 2$

11
$1 \quad 3 / 2$

$3 \quad 3$

$3 / 2$

2

$3 / 2$

$9 / 4$

$3 / 2$

$3 / 2 \quad 3 / 2$

$1 \quad 1$

$3 / 2$

$3 / 2$

Fig. 5: For the regular hexagon, there are 10 types of allowed rescalings (up to rotations and reflections) shown in this figure. In total, we get the Eulerian number $A(5,2)=6+6+6+6+6+6+3+3+12+12=66$ rescalings.

\section{The case of the nonnegative Grassmannian}

The next natural step is to extend the structures discussed above to the case of the nonnegative Grassmannian $G r \geq(k, n)$. In other words, let us now allow some subset of Plücker coordinates to be zero, and try to describe possible arrangements of smallest (largest) positive Plücker coordinates.

Many arguments that we used for the positive Grassmannian, will not work for the nonnegative Grassmannian. For example, if some Plücker coordinates are allowed to be zero, then we can no longer conclude from the 3-term Plücker relation that $\Delta_{13} \Delta_{24}>\Delta_{12} \Delta_{34}$. 
Let us describe these structures in the case $k=2$. The combinatorial structure of the nonnegative Grassmannian $G r^{\geq}(2, n)$ is relatively easy. Its positroid cells [Po] are represented by $2 \times n$ matrices $A=$ $\left[v_{1}, \ldots, v_{n}\right], v_{i} \in \mathbb{R}^{2}$, with some (possibly empty) subset of zero columns $v_{i}=0$, and some (cyclically) consecutive columns $v_{r}, v_{r+1}, \ldots, v_{s}$ parallel to each other. One can easily remove the zero columns; and assume that $A$ has no zero columns. Then this combinatorial structure is given by a decomposition of the set $[n]$ into a disjoint union of cyclically consecutive intervals $[n]=B_{1} \cup \ldots \cup B_{r}$. The Plücker coordinate $\Delta_{i j}$ is strictly positive if $i$ and $j$ belong to two different intervals $B_{l}$ 's; and $\Delta_{i j}=0$ if $i$ and $j$ are in the same interval.

The following result can be deduced from the results of Section 3 .

Theorem 8.1 Maximal arrangements of smallest (largest) positive minors correspond to triangulations (thrackles) on the $r$ vertices $1, \ldots, r$. Whenever a triangulation (thrackle) contains an edge $(a, b)$, the corresponding arrangement contains all Plücker coordinates $\Delta_{i j}$, for $i \in B_{a}$ and $j \in B_{b}$.

We can think that vertices $1, \ldots, r$ of a triangulation (thrackle) $G$ have the multiplicities $n_{a}=\left|B_{a}\right|$. The total sum of the multiplicites should be $\sum n_{a}=n$. The number of minors in the corresponding arrangement of smallest (largest) minors equals the following sum over edges $(a, b)$ of $G$

$$
\sum_{(a b) \in E(G)} n_{a} n_{b}
$$

Remark that it is no longer true that all maximal (by containment) arrangements of smallest (or largest) equal minors contain the same number of minors.

The maximal possible number of equal minors in such arrangements is achieved in the case when we subdivide $[n]$ into 3 consecutive intervals $[n]=B_{1} \cup B_{2} \cup B_{3}$ with sizes $\left|B_{a}\right|$ as close as possible to $n / 3$. In this case, all non-zero minors will be equal to each other.

Theorem 8.2 The maximal size of an arrangement of smallest minors or largest minors in $G r \geq(2, n)$ is

$$
\left\{\begin{array}{cl}
3 m^{2} & \text { if } n=3 m \\
m(3 m+2) & \text { if } n=3 m+1 \\
(m+1)(3 m+1) & \text { if } n=3 m+2
\end{array}\right.
$$

The first case corresponds to a subdivision of $[n]$ into three intervals of sizes $m, m, m$; the second case corresponds to intervals of sizes $m, m, m+1$; and the third case corresponds to intervals of sizes $m, m+1, m+1$.

\section{Final remark}

Skandera's inequalities [Sk] for products of minors discussed in Section 5 are related to monomial positivity and Schur positivity of expressions in terms of the Schur functions the form $s_{\lambda} s_{\mu}-s_{\nu} s_{\kappa}$. In [LPP], several Schur positivity conjectures of this form were proved, including Fomin-Fulton-Li-Poon's conjecture $[\overline{F F L P}]$ and Okounkov's conjecture $[\mathrm{Ok}]$. Schur functions were shown to satisfy a certain Schur-log-concavity property. In a sense, Theorems 4.3 and 4.4 are also manifestations of a similar logconcavity principle. There are seem to be many parallels between the current work on arrangements of equal minors and constructions from [LPP]. We plan to investigate this link in the future. This might lead to more general Schur positivity results. 


\section{References}

[DKK] V. I. Danilov, A. V. Karzanov, G. A. Koshevoy: On maximal weakly separated setsystems, Journal of Algebraic Combinatorics 32 (2010), 497-531.

[FFJM] M. FARber, M. FAUlK, C. R. Johnson, E. MARZIOn: Equal entries in totally positive matrices, arXiv:1309.4186, 2013.

[FRS] M. FARBER, S. RAY, S. SMORODINSKY: On totally positive matrices and geometric incidences, arXiv:1309.45422013, 2013.

[FFLP] S. Fomin, W. Fulton, C.-K. Li AND Y.-T. Poon: Eigenvalues, singular values, and Littlewood-Richardson coefficients, American Journal of Mathematics 127 (2005), 101-127.

[FZ] S. Fomin, A. Zelevinsky: Cluster algebras I: Foundations, J. Amer. Math. Soc. 15 (2002), 497-529.

[GK] F. Gantmacher, M. Krein: Sur les matrices oscillatores, CR Acad. Sci. Paris 201 (1935).

[LP] T. Lam, A. Postnikov: Alcoved Polytopes I, Discrete \& Computational Geometry 38 no. 3 (2007), 453-478.

[LPP] T. Lam, A. Postnikov, P. Pylyavskyy: Schur positivity and Schur log-concavity, American Journal of Mathematics 129 (2007), 1611-1622.

[LS] K. LEE, R. SCHIFFLER: Positivity for cluster algebras, arXiv:1306:2415.

[Lu1] G. LuszTig: Total positivity in reductive groups, Lie Theory and Grometry, Progr. in Math., vol. 123, Birkhauser, Boston, 1994, pp. 531-568.

[Lu2] G. LuszTIG: Total positivity in partial flag manifolds, Representation Theory 2 (1998), 70-78.

[LZ] B. Leclerc, A. Zelevinsky: Quasicommuting families of quantum Pluc̈ker coordinates, American Mathematical Society Translations, Ser. 2 181, 1998.

[Ok] A. OKOUNKOV: Log-concavity of multiplicities with applications to characters of $U(\infty), A d$ vances in Mathematics 127 (1997), no. 2, 258-282.

[OPS] S. OH, A. Postnikov, D. Speyer: Weak separation and plabic graphs, arXiv: 1109.4434.

[Po] A. Postnikov: Total positivity, Grassmannians, and networks, arXiv:math. C0/0609764.

[Sc] J. ScOTT: Grassmannians and cluster algebras, Proc. London Math. Soc. 92 (2006) no. 2, 345380 .

[Sch] I. J. SchoenberG: Über variationsvermindernde lineare transformationen, Math. Z.32 (1930), $321-322$.

[Sk] M. SKANDERA: Inequalities in products of minors of totally nonnegative matrices, Journal of Algebraic Combinatorics 20 (2004), no. 2, 195-211.

[St] B. StuRmfels: Gröbner bases and convex polytopes, University Lecture Series, 8. American Mathematical Society, Providence, RI, 1996. 